Itinéraires Itinéraires

Littérature, textes, cultures

\title{
Corps à corps en 2007 : Nicolas Sarkozy face à Ségolène Royal
}

\section{Hugues de Chanay}

\section{(2) OpenEdition}

1 Journals

Édition électronique

URL : http://journals.openedition.org/itineraires/341

DOI : 10.4000/itineraires.341

ISSN : 2427-920X

Éditeur

Pléiade

\section{Édition imprimée}

Date de publication : 1 mai 2009

Pagination : $61-80$

ISBN : 978-2-296-08444-5

ISSN : $2100-1340$

\section{Référence électronique}

Hugues de Chanay, «Corps à corps en 2007 : Nicolas Sarkozy face à Ségolène Royal », Itinéraires [En ligne], 2009-1 | 2009, mis en ligne le 11 juin 2014, consulté le 02 juillet 2020. URL : http://

journals.openedition.org/itineraires/341 ; DOI : https://doi.org/10.4000/itineraires.341

Ce document a été généré automatiquement le 2 juillet 2020.

\section{(c) (†) $\ominus$}

Itinéraires est mis à disposition selon les termes de la licence Creative Commons Attribution - Pas d'Utilisation Commerciale - Pas de Modification 4.0 International. 


\title{
Corps à corps en 2007 : Nicolas Sarkozy face à Ségolène Royal
}

\author{
Hugues de Chanay
}

1 La retransmission télévisée des débats politiques permet au chercheur d'avoir un accès relativement fin aux dimensions somatiques de la communication politique, entendons par là l'ensemble des aspects que l'on peut appeler à la suite de Cosnier (Cosnier et al. 1982) « voco-mimo-posturo-gestuels », et que l'on a trop longtemps considérés comme des aspects accessoires du discours-ainsi qu'en témoignent les termes de "paraverbal », souvent utilisé pour désigner les données prosodiques, ainsi que de "non verbal», pour désigner les aspects kinésiques de la communication (ce qui suggère, soit qu'ils n'ont pas de vertu propre et sont incapables de véhiculer des contenus fins, soit encore, plus gravement, que le Verbe peut très bien s'en dispenser, comme l'atteste l'habitude de s'en tenir à ses transcodages écrits, dont on mesure souvent très mal ce qu'ils lui font perdre).

2 Dans la conception que nous voudrions illustrer ici, le corps n'est pas un simple diffuseur du Verbe, lequel s'y ferait chair à la faveur de la présence physique de ses émetteurs, mais pourrait tout aussi bien s'inscrire ailleurs sans perte (par exemple, pour le débat du 2 mai 2007 entre Ségolène Royal et Nicolas Sarkozy qui nous occupera ici $^{1}$, dans sa transcription faite par Libération, qui était disponible dès le lendemain sur Internet $\left.{ }^{2}\right)$. Au contraire, la présence des débatteurs en chair et en os, outre qu'elle contribue à assurer leur identification (leur " image ») de manière bien plus efficace et parfois périlleuse - que ne le ferait tout autre type de participation, délivre un apport communicatif propre, non seulement parce que les corps constituent les supports d'inscription non neutres sur lesquels le verbal, d'une certaine manière, s'écrit et s'actualise (c'est l'actio de la rhétorique classique ${ }^{3}$ ), mais encore parce qu'ils écrivent eux-mêmes au sein de la partition communicative globale leurs propres lignes, non moins essentielles et différenciées que ne le seraient celles d'un ou même de plusieurs instruments de musique appelés à figurer dans un effectif orchestral. On se situera donc dans la lignée des approches « holistiques » (Kraft et Dausendschön 2001) de la communication orale, pour lesquelles elle doit être envisagée comme une totalité 
verbo-somatique (le «totexte » : Cosnier et al. 1982 ; Cosnier et al. 1984; Cosnier 2000), certains préconisant même de la rebaptiser "somatic communication" (Scollon et Scollon 1995), avec pour objectif tout à fait clair d'évacuer les présupposés verbocentriques qui grèvent souvent l'analyse de discours et de pointer, en contrepartie, le rôle central du corps.

Ce corps, dans le cas du débat qui nous intéresse ici, est en outre mis en scène, et pour tout dire spectacularisé, à l'intention de l'immense auditoire des téléspectateurs appelés quelques jours plus tard à voter pour élire leur président ou leur présidente : ce qui a pour conséquence que, comme sur la scène d'un théâtre, tout du corps est sémiotisé, jusqu'aux indices non communicatifs - rides, sueur, tics, agitation, raideur... - qui ordinairement ne sont pas destinés à être assumés comme ingrédients sémiotiques pertinents dans l'interaction, mais qui en l'occurrence ne manqueront pas d'être portés au crédit ou au discrédit du candidat. Son corps ne montre pas seulement qui il est (on peut à cet égard parler d'une véritable "signature voco-corporelle » qui nous compose une identité qui est, c'est le cas de le dire, chevillée au corps, et dont on ne se débarrasse jamais), mais surtout, plus insidieusement, semble montrer quel il est, et ainsi permettre, non pas seulement de l'identifier et de le reconnaître, mais, plus substantiellement, de le connaître avec une sorte d'évidence immédiate en voyant incarnées, dans les postures (leur tonus, leur ouverture, leur réactivité), dans les mimiques, dans l'ensemble de la gestuelle (y compris vocale), les différentes qualités dont le candidat essaie de faire preuve (ou plutôt de « faire montre »). C'est dire que le corps ne convoie pas seulement du logos, mais qu'il est aussi l'un des vecteurs fondamentaux de ce que la tradition rhétorique appelle l'éthos, cette image de soi que l'orateur construit en public à travers son discours et par son discours afin de soutenir son argumentation en la rendant crédible.

La notion d'éthos dont je ferai usage ici comporte quelques petits réaménagements par rapport à celle dont il est habituellement fait usage, dont on peut faire remonter la définition à Aristote (essentiellement dans deux passages de la Rhétorique : I, 1356a et II, 1377b). Je les envisage brièvement ${ }^{4}$. Il s'agit d'abord de la place de l'éthos dans la chaîne d'élaboration discursive que déploient les traditionnelles parties de la rhétorique: Aristote loge l'éthos dans l'inventio, en raison de son statut de preuve, mais il est évident que ses marqueurs se retrouvent transversalement aussi bien dans la dispositio que dans l'elocutio, et, last but not least, dans l'actio - véritable prestation discursive par laquelle, tel un acteur de théâtre, l'orateur tâche de performer au mieux son discours en usant de tous les moyens (voix et gestes) que lui offre son corps. Palette toute personnelle, qu'il convient de bien connaître si l'on veut en maîtriser les effets; aussi ces assises corporelles de l'éthos, dans le cas de la communication politique, sont-elles la plupart du temps extrêmement travaillées, et même s'il entre une part d'improvisation dans les débats (en particulier du fait de leur caractère interactif), la référence au théâtre est loin d'être une simple métaphore: sur les plateaux de télévision comme sur les scènes, on a affaire à des candidats qui se sont rodé un comportement corporel chargé de donner une lisibilité optimale à ce qu'ils veulent paraître. Ils n'y parviennent d'ailleurs pas toujours: d'où l'utilité d'un second réaménagement, concernant la nature de l'éthos: alors qu'Aristote l'envisage exclusivement comme image favorable, il est utile d'envisager la possibilité d'un éthos défavorable ${ }^{5}$, d'autant plus que les qualités dont se composent ces éthè sont loin d'être aussi bien définies que ne le laisse supposer le célèbre trio aristotélicien (compétence, vertu, bienveillance), et surtout sont axiologiquement réversibles, comme l'a montré 
Charaudeau (2005): intelligence/ruse, compassion/faiblesse, culture/frivolité, fermeté/dureté, souplesse/mollesse, esprit fédérateur/démagogique, sérieux/austérité, etc., autant de couples ambivalents - la question de leur inventaire restant par ailleurs ouverte. Enfin, dernière précision: alors que l'éthos oratoire est traditionnellement envisagé sous un angle exclusivement monologal, il convient au contraire, afin de tenir compte de la nature même de ce qu'est un débat (impliquant plusieurs participants), de l'envisager sous un angle interactif, chaque éthos se définissant de manière dynamique face aux autres éthè, avec une certaine souplesse d'adaptation, dans les limites toutefois d'une stabilité éthique globale de rigueur (faute de laquelle la crédibilité pâtit d'un déplorable effet de girouette). On n'agit pas seulement sur sa propre image, mais aussi, du même coup, sur celle des autres - et réciproquement. Dans le cas d'un face-àface, on peut ainsi dire que les éthè des deux partenaires résultent de manière complexe de "la confrontation entre l'image projetée (ou revendiquée) par A, et l'image attribuée à A par B » (Kerbrat-Orecchioni 2000 : 187). Affaire de corps dans l' actio, l'éthos devient donc, dans l'interaction, affaire de corps à corps.

Loin de moi la prétention de traiter exhaustivement les problèmes soulevés par le rôle du corps dans les débats politiques médiatiques. Pour limiter mon propos, je l'aborderai sous l'angle particulier de son extrême plasticité énonciative, ce qui me permettra d'envisager la « corpographèse » sous un double aspect. D'une part, ce qui du discours et de l'interaction s'écrit dans le corps: c'est-à-dire l'enracinement somatique de l'énonciation, aux sources de la gestion des modalités discursives et de l'engagement illocutoire. D'autre part, et complémentairement, ce que le corps écrit dans (et de) l'interaction : c'est-à-dire la gestion dialogale de la configuration des circuits d'adresse, et le déploiement et l'attribution dialogique des points de vue, en une «écriture corporelle » très polyphonique dont on verra chemin faisant comment elle contribue, par petites touches répétées et insistantes, à construire à chacun un éthos - l'éthos, ultime corpographèse peut-être de toute interaction.

\section{L'enracinement somatique de l'énonciation}

6 Je reprendrai ici les vues développées par Berrendonner (1981), qui les avait présentées sous le titre provocateur de "Quand dire, c'est ne rien faire » (partie III de l'ouvrage cité), qui prend évidemment le contre-pied du titre français de l'ouvrage bien connu d'Austin, Quand dire c'est faire, considéré comme l'un des principaux fondateurs de la théorie des actes de langage. Risquons un résumé lapidaire de la thèse de Berrendonner: à proprement parler, il n'y a pas d'actes de langage (et plus exactement: pas d'actes illocutoires). Certes, il y a bien des actes que nous pouvons effectuer en parlant - asserter, promettre, questionner, ordonner... - mais ce n'est pas le langage qui les effectue. Pour Berrendonner, le langage ne peut que représenter, mais il ne peut rien faire. Si l'on peut faire quelque chose grâce à lui (des actes de langage), ce n'est qu'indirectement, "secondairement" (Kerbrat-Orecchioni 2004 : 32). C'est dire que, dans tous les cas, la teneur pragmatique de ces actes, leur statut même d'actes, ne tient en rien aux énoncés - et en particulier, ne tient en rien aux " formes de phrase " (déclarative, jussive, interrogative, éventuellement optative) auxquelles on rattache habituellement les actes de langages dits littéraux ou directs. D'où vient-elle alors, si ce n'est pas des énoncés? Réponse : de l'énonciation. C'est-à-dire, pour Berrendonner, du 
corps. Précisons : d'une certaine utilisation du corps, qu'il qualifie de "gesticulation » (1981:121).

7 Cette gesticulation, c'est la profération, qui correspond à l'acte locutoire d'Austin, seul "acte de langage " que Berrendonner soit prêt à lui concéder- «parler», dit-il, "constitue bien un acte particulier, à condition de ne voir dans la parole que l'accomplissement d'une gesticulation phonatoire et rythmique, révélatrice d'une activité de combinaison syntagmatique» (id.: 81). C'est cet acte d'énonciation fondateur, enraciné dans le corps ou même fusionné avec lui en tant que comportement, et insistons-y, unique comportement à pouvoir être labellisé « acte de langage ", qui est à la source des valeurs illocutoires, toutes indirectes, et dépendantes de cette incarnation primitive :

À quoi voit-on en effet qu'un locuteur s'empare d'une proposition, l'assume, et la soumet à la validation d'autrui ? Essentiellement, au simple fait, parfaitement locutoire, qu'il l'énonce, c'est-à-dire la performe, prononce le signifiant à elle associé, bref, l'actualise [...]. Je propose donc de considérer que la valeur primitivement «constative» ou «assertive » de tout énoncé est portée par l'acte locutoire d'actualisation de cet énoncé, considéré comme symptôme gestuel. [...] Et ce qui se montre, dans le geste locutoire d'énonciation, n'est autre que ce que l'on appelle ailleurs l'« assertion », c'est-à-dire le risque même de la prise de parole. (Id. : 121-122)

8 Je n'entrerai pas ici dans le débat, que je me contenterai de signaler, du caractère fondamental de l'assertion par rapport aux autres valeurs illocutoires-qui, selon Berrendonner, peuvent toutes en être dérivées: interroger, par exemple, serait primitivement asserter qu'on ne sait pas quelque chose, ce qui met l'interlocuteur en posture de fournir le renseignement manquant, s'il le détient (id.: 141-171, spéc. $\left.168-171^{6}\right)$; il reconnait toutefois que les choses sont plus complexes avec les énoncés jussifs (id. : 146) - même si les présupposés pragmatiques des directifs permettent de récupérer dans tous les cas des assertions associées de type « je désire que... ». Mais ce qui importe ici, c'est que, quelles que soient les solutions descriptives apportées au problème de la genèse de telle ou telle valeur illocutoire, cette valeur ne sera véritablement perçue comme opérante - c'est-à-dire ne pourra prendre valeur d'acte que si elle est portée par une énonciation physiquement ancrée, convaincante parce que convaincue et plus exactement se montrant ${ }^{7}$ comme telle. Dans l'engagement physique, la conviction est saisie symptomatiquement dans le hic et nunc en acte d'un comportement dont la réalité ne peut qu'être constatée - infiniment plus solide qu'un dire dont la vérité pourrait être contestée. En un sens, ce qui s'énonce dans le corps est plus proche de l'écrit, du fait de sa matérialité qui le rapproche d'un objet, bien difficile à dissiper, plutôt que de la parole, par définition vulnérable à des épreuves de véridiction capables de l'annuler.

9 Naturellement, les modalités gesticulatoires de cet engagement sont extrêmement diversifiées, et tous les locuteurs ne disposent d'ailleurs pas des mêmes moyens en la matière, certains étant nettement plus mobiles que d'autres (notons au passage qu'on voit déjà pointer une facette de l'éthos, ambivalente comme on pouvait s'y attendre, selon qu'on percevra ces locuteurs comme vifs ou comme agités; ou, sur le versant placide, comme apathiques ou comme posés).

10 Dans les pages qui suivent, je vais me concentrer sur le seul personnage de NS, beaucoup plus diversifié posturalement, gestuellement, mimiquement, vocalement que $\mathrm{SR}$, laquelle - du moins dans ce débat-ci, car on peut l'observer très différente dans un 
autre débat pourtant temporellement très proche, également entre les deux tours mais face à François Bayrou ${ }^{8}$ - maintient peu ou prou la même pose tout au long du débat, c'est-à-dire tête et buste droits, orientés quasi exclusivement sur son interlocuteur principal, les yeux rivés sur lui, le plus souvent sans mouvements de sourcils ni sourire (ce que l'on peut interpréter comme une stratégie pour s'abstenir visiblement, dans ce contexte de confrontation publique, de toute démonstration empathique), avec pour seule gestuelle une rythmique de scansion, de la tête surtout, moins souvent des mains qui restent plus volontiers à plat sur la table, tout ceci à l'exception notable d'un geste de l'index, après deux bonnes heures de débat, dont nous aurons à reparler: physiquement, nous avons donc l'image et presque la démonstration (en tout cas, la monstration) d'une candidate qui campe sur sa position, tient bon, fait front, et projette dans son maintien où rien - œil, tête, buste, ni même voix - ne dévie, toute la détermination, la solidité (mais on pourrait dire aussi : la rigidité), la vigilance de qui garde le cap. Face à cette incarnation monologique, NS va au contraire écrire corporellement une partition multiplement polyphonique.

\section{Une corpographèse polyphonique}

11 À l'envisager sous un angle purement monologal, NS est, comme chacun d'entre nous, un seul locuteur, une seule voix, un seul corps : mais sous cette apparente unicité les théories du dialogisme (dans la lignée de Bakhtine) et de la polyphonie (dans celle de Bally et de Ducrot) ${ }^{9}$ ont depuis longtemps débusqué une pluralité de discours. Cela étant, l'histoire de ces théories fait, comme ailleurs en linguistique, la part belle à l'écrit, et, sans être inexistantes, les applications à des corpus multimodaux sont encore jeunes et fragmentaires ${ }^{10}$. Le geste en particulier semble le parent pauvre des études polyphoniques ; toutefois, ce délaissement apparent est en partie illusoire, du fait que les analystes du geste qui s'occupent d'hétérogénéité énonciative (en particulier Bouvet 2001, Calbris 2003 et 2008) n'explicitent pas, ou peu, la nature dialogique/ polyphonique des phénomènes qu'elles décrivent - elles ne les en décrivent pas moins, et dans ce qui suit je m'inspire en partie de leurs travaux. Sans prétendre bien sûr épuiser le sujet, je me concentrerai sur trois fonctions des "gesticulations" polyphoniques sarkozyennes qui me paraissent caractéristiques du personnage et récurrentes tout au long de ce débat, à savoir : asserter ; confronter ; répartir. Ces trois fonctions sont bien sûr intriquées dans la plupart des séquences, et je ne les sépare que pour la commodité de l'exposé.

\section{Asserter}

12 Comme on l'a suggéré plus haut, la valeur assertive, à suivre Berrendonner, tient moins à la forme de phrase qu'à l'engagement physique manifesté lors de la profération, qui fait que les propositions se trouvent investies par le locuteur qui prend le risque de s'y associer physiquement et de les «occuper». Or investies, elles peuvent l'être de plusieurs façons.

13 Le cas le plus simple est celui où le propos asserté exprime une position défendue par le locuteur. Dans ce cas de figure, la tendance de NS va majoritairement et classiquement au martèlement, par hochement répété de la tête sur l'axe vertical ${ }^{11}$ et/ou par les mouvements des mains, qui s'abaissent et se relèvent rythmiquement, seules ou en 
paire, doigts tendus ou au contraire regroupés en poings serrés, capables de s'abattre au centre pour marquer le pic d'engagement argumentatif de l'énoncé verbal en cours. Ces « beats " gestuels s'accompagnent généralement de pics prosodiques (en fréquence et en intensité), ce qui nous a amené par ailleurs à parler pour NS, étant donné la régularité chez lui de ce type de coïncidence, de "convergence multimodale " au service d'un éthos de /fermeté/ (Constantin de Chanay et Kerbrat-Orecchioni 2007). Dans l'extrait suivant les flèches descendantes marquent les battements des deux mains, pour le premier, puis de la main droite seule (je néglige tous les autres gestes), produisant un très fort effet d'accord avec les propositions ainsi scandées, l'accumulation finale constituant la loi énoncée en conclusion présentée comme impossible à démolir tant il s'y accumule de poids ${ }^{12}$ :

(00.38.15) NS : [...] qu'est-ce que j'propose (.) une autre stratégie (.) la stratégie qui a marché (.) partout $\downarrow$ (.) vous citez souvent des démocraties du Nord de l'Europe (.) c'est ç'qu'i font $\downarrow$ (.) vous avez : votre ami Blair (.) eu :h au Royaume-Uni (.) c'est ç'qu'il a fait $\downarrow$ (.) monsieur Zapatero (.) c'est ç'qu'il a fait $\downarrow$ (.) le $\downarrow$ travail $\downarrow$ des uns $\downarrow$ crée le $\downarrow$ travail $\downarrow$ des autres $\downarrow$

On peut trouver des variantes complexes de ces battements, qui peuvent se combiner avec une métaphore de l'avancée rapide et régulière (le progrès) : les deux poings fermés ramenés au centre à hauteur de plexus solaire sur le mot « moi » (signalé par les deux astérisques dans l'extrait ci-après) font des rapides va-et-vient rotatifs sur l'axe sagittal (j'indique par des flèches ascendantes et descendantes les points d'inversion du mouvement, les premières correspondant aux temps forts de la scansion) :

(00.29.30) NS : [...] ce que je souhaite moi** (.) c'est li $\uparrow$ bérer $\downarrow$ la possibilité $\uparrow$ d'tra $\downarrow$ vailler $\uparrow$ en $\downarrow$ Fran $\uparrow$ ce

Nul doute, visuellement parlant, que l'on s'acheminera alors vers un avenir radieux dont l'auditoire sera le bénéficiaire, puisque c'est dans ces deux directions, métaphoriquement pour le premier et réellement pour le second, que roulent les mains unies avec une fluidité tout euphorique, et comme partant du cœur.

L'investissement physique du locuteur, toutefois, ne signale pas toujours comme dans ces deux exemples une prise en charge personnelle - l'assertion produite peut être reversée à un autre personnage, campé en direct sur le devant de la scène, mais revêtement tout éphémère du locuteur qui en garde le contrôle dans des coulisses plus ou moins visibles. J'envisage successivement les cas de l'antiphrase, puis du discours représenté (DR).

17 Avec l'antiphrase nous entrons en terre polyphonique : un point de vue est asserté explicitement, pour ainsi dire en devanture d'énoncé, qui ne correspond pas au point de vue qui doit être décodé réellement, lequel est dans les cas les plus typiques l'inverse $\mathrm{du}$ premier. Berrendonner présente ce phénomène comme un "paradoxe argumentatif» (1981: 222), précisément une dissociation entre l'énonciation corporellement montrée (l'engagement physique qui produit l'assertion), et la représentation argumentative véhiculée par l'énoncé, ou l'ensemble d'énoncés qui permet de repérer l'orientation textuellement pertinente : ainsi, «ce que dit l'énoncé est le contraire de ce que dit l'énonciation » (ibid.). Entre les deux, il faut donc choisir, et l'on sait que cela peut être dans certains cas très délicat. Toutefois dans un débat politique public, on n'a pas avantage à présenter des dilemmes difficiles à trancher, qui obscurciraient les positions - chose évidemment contre-productive. Malgré cela, NS manie assez souvent l'antiphrase, dont les bénéfices éthiques (pour autant que le procédé soit bien dosé) sont assez considérables, et qui plus est immédiats: en 
représentant la position adverse, on fait preuve d'ouverture d'esprit, tout en se plaçant simultanément en position supérieure, puisqu'on la discrédite ; cette hiérarchie a en outre un caractère d'évidence, puisqu'elle n'est pas dite, et qu'on laisse à la charge de l'auditoire de trancher le dilemme (en montrant qu'il pourra le faire sans aucune difficulté); enfin, on peut engranger une image de finesse à jouer pour le public ce double jeu, l'humour de l'énonciation non sérieuse faisant souffler un petit vent de fraîcheur dans des débats parfois un peu plombés. Reste néanmoins à se faire comprendre. On peut constater à cet égard que l'investissement corporel d'assertion de NS en cas d'antiphrase n'est pas le même que dans le cas des propos littéralement assumés, comme on peut le voir dans l'extrait suivant :

(01.06.15)

SR : [...] la relance de la croissance économique va aussi permettre des cotisations supplémentaires (.) moi je crois (.)

[que la croissance éc-\&

NS : [(ben ça) donc vous créez une taxe\&\&

$\mathrm{SR}: \&[j \mathrm{je}$ crois que la c- attendez laissez-moi

NS : \&\&[sans dire aux Français le montant de cette taxe (.) et l'espérance de recettes

SR : oui parce que [je di :s\&

NS : [ah ben (d'accord)

SR : \&aux Françai :s

NS : ben avec ça on est [tranquilles\&

$\mathrm{SR}:$ : [parce que

NS : \&pour l'équilibre de nos régimes de retraites

SR : parfaitement

NS : ah oui

SR: on est tranquilles pour l'équilibre de nos régimes de retraite parce que moi (.)

au moins (.) j'ai une recette

Durant la séquence antiphrastique, ci-dessus en gras, c'est NS qui est à l'écran, ce qui permet d'observer, non pas comme précédemment des battements ou des hochements sur l'axe vertical, mais des oscillations de la tête sur l'axe latéral - la tête balançant d'une inclinaison à l'autre sans fixer aucune approbation de ce qui est physiquement considéré sous divers angles. Le point de vue assumé est donc de type /on est loin d'être tranquille/: le contraire de ce qui est dit, mais en convergence avec ce qui est montré. Le point de vue discrédité n'en a pas moins été asserté par une sorte de personnage fictif que NS a comme projeté au-devant de lui-même, par la profération locutoire, tout en marquant une distanciation claire grâce aux mouvements de tête ; et c'est l'existence de cette assertion qui permet à SR d'enchaîner par un adverbe confirmatif (qui comme réponse à une antiphrase prend une valeur contestataire) puis de reprendre mot pour mot le propos, cette fois-ci littéralement - et physiquement assumé.

Passons rapidement au DR, en terre dialogique cette fois. Pour bien faire il conviendrait d'envisager deux cas, selon que NS se montre en accord ou en désaccord avec les propos rapportés. Dans le premier cas, l'assomption physique reproduite tend-cela semble assez inévitable: nous n'avons qu'un seul corps - à fusionner avec celle qui exhibe l'investissement propre du locuteur dans le hic et nunc interactif, ce qui, gestuellement, revient au premier cas d'assertion simple (et du point de vue dialogique, on est dans le cas de ce que Perrin [2004] nomme "discours modal»: les propos rapportés ne sont pas simplement mentionnés, mais argumentativement repris). Dans le second cas, toujours pour la même raison (on parle avec son corps, unique), il convient au contraire de se démarquer physiquement de l'assertion reproduite. Dans l'extrait suivant je vais 
coder comme plus haut les battements assertifs par des flèches simples descendantes $(\downarrow)$, mais aussi les regards vers AC et PPDA par une flèche simple orientée à gauche $(\leftarrow)$, le retour vers SR par la même flèche orientée à droite $(\rightarrow)$, et j'emploierai des flèches doubles pour les avancées $(\Rightarrow)$ ou les reculs $(<=)$ du buste. Ce qui donne :

$(00.30 .20)$ NS : $[. ..] \rightarrow<=$ vous dites on paye trop aux entreprises (.) mais est-ce que vous dites $=>$ aux Français (.) que les 35 heures ça coûte (.) dix $\downarrow$-sept $\downarrow$ mi $\downarrow$ liards $\downarrow$

d'euros $\downarrow$ (.) chaque année $\downarrow$ (.) $\leftarrow$ pour empêcher les gens d'travailler $/ \downarrow$

Le schéma est clair : le désaccord avec ce que SR dit est mis en scène par le recul du buste, et l'accord avec ce qu'elle ne dit pas, mais devrait dire, par le mouvement inverse du penchement en avant, qui permet de réintroduire les battements assertifs sur l'argument choc du coût (présenté comme exorbitant), pour clore en beauté sur la prise à témoin des journalistes, appelés à constater de concert avec NS l'absurdité de la dépense. On voit que cet exemple illustre également les deux autres fonctions que j'ai annoncées plus haut: confronter les points de vue, et répartir les positions sur les participants - deux fonctions auxquelles je viens à présent brièvement.

\section{Confronter}

Nous sommes ici en terre polyphonique, y compris en ce sens précis que les positions confrontées peuvent être purement structurelles-c'est-à-dire indispensables à envisager pour la compréhension des énoncés, mais pas forcément endossées par l'un ou l'autre des participants. Je prendrai l'exemple classique de la négation, intéressante parce qu'elle peut se réaliser linguistiquement, mais aussi gestuellement. Or, comme on l'a souvent remarqué, il n'y a pas forcément correspondance modale entre les deux ordres sémiotiques, ce qui introduit des confrontations de points de vue qui resteraient insoupçonnées si l'on ne considérait que le texte, sans l'accompagnement gesticulatoire qui lui fournit un contrepoint continûment inscrit dans le corps. Soient d'abord, sans grande surprise, des battements assertifs marquant physiquement l'accord avec des énoncés à modalité linguistique négative (même codage que ci-dessus, à ceci près que je duplique la double flèche pour marquer une avancée du buste plus forte; en gras, les passages pertinents pour mon propos):

$(00.28 .44)$ NS : [...] $\rightarrow$ il y a $\Rightarrow$ dix $\downarrow$ pays en Europe (.) qui ont le plein-emploi $($.) $\neg$ dix $\downarrow$ (.) (y en a :) cinq vraiment très forts (.) je pense au Danemark à la Suède à l'Irlande $\rightarrow$ au Royaume-Uni dans une certaine façon l'Espagne $()<.=$ ça devrait vous frapper $\downarrow$ madame Royal (.) y en a pas un seul d'entre eux $\downarrow$ > qui a fait le partage du temps de travail (.) y en a pas un seul d'entre eux $\downarrow$ => qui a fait les $\rightarrow 35$ heures $\downarrow$ monsieur Zapatero qui est venu dans le meeting de Toulouse que vous avez tenu (.) =>=> m'a dit $\downarrow$ (.) à Madrid (.) que <= jamais il ne ferait $\downarrow$ les $\leftarrow$ 35 heures (.) en Espagne (.) qu'il ne voulait pas porter atteinte à la compétitivité $\rightarrow$ des entreprises

Ce marquage revient, en somme, à asserter que l'on nie ${ }^{13}$ - à assumer physiquement la négation dite. La combinatoire avec les mouvements d'avancée et de recul marque quant à elle une opposition approbation/désapprobation en convergence globale avec les négations assertées, assez subtilement parfois : ainsi le recul lors de l'introduction des propos de Zapatero doit sans doute être interprété comme une convergence dans le refus d'envisager l'idée des 35 heures (cas évoqué plus haut du « discours modal », avec accord entre l'argumentation du locuteur actuel et les propos du DR). 
23 Le cas inverse est également bien attesté : une négation gestuelle vient, non pas annuler un énoncé linguistiquement assertif, mais y associer un point de vue en confrontation. Deux exemples entre mille (je marque le pivotement latéral de la tête par une flèche bi-orientée, et je mets en gras le segment pendant lequel il se poursuit) :

(01.27.34) NS : [...] alors s'agissant d'ailleurs de l'environnement (.) je veux dire prenant mes responsabilités que si je suis président de la République je confirmerai $\leftrightarrow$ le choix du nucléaire

(00.08.44) NS: [...] donc sur le bilan en matière de violences (.) en matière de délinquance (.) avec le même $\downarrow$ appareil statistique $\downarrow$ madame Royal $\leftrightarrow$ le même (.) $\leftrightarrow$ rien n'a changé c'est le même à l'époque de Daniel Vaillant et c'est l'même maintenant

Dans le premier exemple, la négation - d'autant plus intéressante qu'elle intervient immédiatement après un promissif en modalité assertive - ajoute un point de vue que l'on pourrait paraphraser ainsi : /il existe une position que je connais, qui consisterait à revenir sur le choix du nucléaire; et je refuse cette position/. Dans le second, la négation sur le segment «le même " projette un point du type /pas un autre/ qui se trouvera, après une courte pause, explicité par "rien n'a changé »: preuve que le discours s'élabore dans une planification multilinéaire où le corps généralement anticipe la parole qui va s'y inscrire.

\section{Répartir}

On l'a déjà remarqué au travers des différents exemples que nous avons énumérés, le regard de NS est très mobile, contrairement à celui de SR qui cantonne AC et PPDA dans leur strict (et assez minimal pour ce qui est de ce débat) rôle d'animateurs, ne se tournant vers eux que par deux fois pour solliciter la conservation de la parole d'un bref «Vous permettez ? ». NS, au contraire, crée des coalitions par le regard, enrôle les journalistes comme cautions passives pour des points de vue qu'il défend, et qu'il enrichit de leur autorité : car qui ne dit mot consent. Ainsi, en tant que connaisseurs de l'actualité et de l'histoire récente, AC et PPDA simplement sélectionnés d'un regard sont enrôlés comme caution légitimante pour l'argument en faveur de la réduction des emplois publics - sorte d'argument ad hominem puisqu'il fait intervenir contre SR quelqu'un qui peut être tenu pour une figure emblématique de son propre camp :

(00.18.58) NS : [...] bon (.) avec c'que j'propose madame (.) nous reviendrons aux effectifs de la fonction publique en 1992 époque où François Mitterand $(.) \leftarrow$ était président d'la République $\rightarrow$ (.) je ne sache pas qu'à l'époque (.) la France était (.) sous-administrée

L'attribution de positions peut se faire également, bien entendu, par pointage. Dans l'extrait suivant, qui précède immédiatement la célèbre colère de $S R$, je code par ** un geste de désignation de l'interlocutrice par la paume ouverte en supination avancée dans l'axe sagittal, et par * un geste d'autodésignation par le pouce pointé en direction $\mathrm{du}$ visage, et je mets en gras les segments sur lesquels ils portent :

(01.54.30) NS : [...] la preuve de ma bonne foi (.) et la certitude de la réalisation de cette promesse (.) ça sera le droit opposable (.) et la [capacité (.)\&

SR : [(mais (.) est-ce)

NS : \&d'aller devant un tribunal pour faire-valoir ses [droits (.) vous voyez madame

(.)\&

$\mathrm{SR}:[$ (que vous)

NS : \&c'est ni ridicule (.) ni accessoire (.) [c'est peut-être\& 
(PPDA ?) : [(alors)

NS : \&même ce qui fait la différence (.) entre ${ }^{* *}$ la vieille politique (.) et * la

politique [moderne

PPDA : [alors vous avez réussi [tous les deux [la prouesse

AC : [(xx alors j'ai un vous voulez dire)

SR : [attendez (.) j'ai quelque chose à dire attributions aux participants de points de vue ou plus généralement de positions qu'ils sont censés avoir préalablement, et/ou acquérir durablement : à ce titre, on peut les considérer comme dialogiques. Reste à dire - ce qui n'est pas une nouveauté - que le corps configure les rôles interactifs eux-mêmes, au niveau dialogal ${ }^{14}$ cette fois-ci. On l'a mentionné plus haut, SR cantonne les journalistes dans leur rôle d'arbitres, et posturalement, organise l'interaction en un face-à-face, voire un duel. En un sens, elle combat. NS, lui, s'adresse à tous, avec un regard mobile qui assure la "maintenance » de la participation de tous à un polylogue. Par contraste, il débat, et même s'ils ne pipent mot, les journalistes sont intégrés à titre de participants ratifiés par une mobilité aussi bien oculaire que posturale. Chose remarquable, s'il y a parfois exclusion, c'est à rencontre de SR, qui se trouve à plusieurs reprises "délocutée in prcesentia », c'est-à-dire qu'en sa présence, NS parle d'elle, mais pas à elle. Témoin ces commentaires de NS pendant la fameuse colère de SR :

(01.58.20) SR : [...] non (.) justement pas

$[($.$) je suis en colère face aux injustices ($.$) face a u(x)$ mensonge(s)

NS: [je ne vois pas pourquoi: (.) madame Royal (.) ose (.) employer le mot

(.) $\neg$ immoral (.) c'est un mot

$\leftarrow$ fo :rt(.)\&

SR : [oui :(.) c'est

NS : \&madame Royal se permet d'employer ce mot (.) parce que j'ai dit (.) que je souhaitais que tous les enfants (.) ayant un handicap soient scolarisés en milieu scolaire (.) entre guillemets normal

Les regards résolument tournés vers AC et PPDA excluent provisoirement SR du circuit interlocutif. Il s'agit déjà d'un cas particulier d'une tendance récurrente de NS dans ce débat à se placer en position non seulement de participant, mais de commentateur. Mais il s'agit aussi d'une stratégie d'exclusion qui projette des éthè radicalement contrastés, d'une SR isolée et rigide, face à un NS fédérateur et flexible. Ces « qualités » auraient pu être axiologisées différemment: le fédérateur pourrait être vu comme démagogue, sa flexibilité comme opportunisme ; quant à la rigidité, elle pourrait être fermeté ou résistance, et l'isolement un refus du compromis. Que les valeurs se soient semble-t-il distribuées autrement, cela suggère - ou plutôt, cela tend à confirmer, car nous ne sommes pas le premier à le relever - que dans un contexte de débat, où représenter des positions autres c'est afficher qu'on sait les prendre en considération, et qui plus est dans un contexte de second tour d'élection présidentielle, où la capacité à gouverner s'accorde peut-être plus immédiatement avec la capacité à fédérer qu'avec l'ardeur à militer, la polyphonie - qui eût peut-être évité à l'altier de se faire percevoir 
comme hautain - est éthiquement plus avantageuse que le maintien d'une ligne unique, fût-elle pure ${ }^{15}$.

\section{Remarques finales}

Cette éloquence polyphonique, nous nous sommes efforcé de le montrer, est en grande partie affaire de gestes, de regards, de postures, qui supportent aussi bien qu'ils remodèlent le discours «linguistique» (au sens restreint) dans la matérialité constamment active du corps qui en est tout à la fois support d'énonciation, et énonciation lui-même avec ses énoncés propres qu'il intègre au discours global (au «to-texte ») : c'est en ces deux sens que nous avons pris le terme de « corpographèse ».

Je suis loin d'en avoir épuisé les vertus heuristiques pour la question de la construction des éthè. Une petite incursion dans le contrastif me permettra pour finir d'en esquisser deux aspects complémentaires et tout aussi fondamentaux l'un que l'autre, le premier illustrant la relative liberté du geste d'inscription, et le second la relative permanence des écrits.

La liberté, d'abord. Lorsqu'avec C. Kerbrat-Orecchioni nous avons analysé un autre débat avec NS, face à T. Ramadan puis à J.-M. Le Pen, nous avons repéré comme l'un des procédés caractéristiques de la rhétorique gestuelle sarkozyenne l'index pointé vers l'interlocuteur (2007: 318 et 326): index fortement accusateur, qui renforce des sommations, lance des défis, et intervient dans chaque cas pour aider NS à prendre l'avantage à des moments cruciaux où le débat devient conflit. Or ce geste n'apparaît pas chez NS dans ce débat-ci ; mieux, c'est chez SR qu'il survient, pendant sa « saine colère »; et mieux encore, NS le lui reproche (alors qu'il fait partie de sa palette habituelle) :

$(01.57 .00)$

$\mathrm{SR}$ : \&et je suis très en colère (.) et les parents et les familles qui vous

[ent-\&

NS : [calmez-vous

SR : \&et les parents $\neq$ [non je ne me calmerai pas

NS : [calmez-vous et ne me montrez pas du doigt avec

[ce cet index\&

$\mathrm{SR}:[$ non (.) si

NS : \&[pointé parce que franchement (.) je je je voudrais vous di- je voudrais

SR : [non (.) non je ne me calmerai pas (.) non je ne me calmerai pas (.)

[je ne me \&

NS : [ben pour\&\&

SR : \&[calmerai pas (.) parce que l'exploita- $\rightarrow$

NS : \&\&[(.) pour être président de la République i faut êt' calme

$\mathrm{SR}: \rightarrow$ non (.) pas quand il y a des injustices

Il est vrai qu'en une minute trente à peine, SR a lancé son index dans l'axe sagittal une bonne quinzaine de fois. À bien y regarder toutefois, il ne s'agit pas tout à fait du même geste que celui qu'incrimine NS : l'index de SR hésite entre la position à la verticale (doigt levé de la leçon de morale) et la position à l'horizontale (pointage accusateur de NS), qu'elle ne maintient jamais; en outre, il est simultanément utilisé à des fins de scansion, ce qui atténue la valeur agressive du geste en y faisant cohabiter le marquage de la conviction personnelle. C'est donc au prix, peut-être pas d'une mauvaise foi, mais tout de même d'une sélection orientée et d'une amplification, que NS peut reconstruire ce geste comme celui du pointage accusateur qui fut si souvent le sien (et, est-il besoin 
de le préciser, toujours dans une version exclusivement horizontale et fermement maintenue) mais dont il se sera cette fois-ci abstenu - la raison invoquée étant que la qualité éthique dont il témoigne, une sorte de pugnacité inflexible, qui pouvait être prisée lorsque NS était ministre de l'Intérieur, ne construit pas l'éthos idéal d'un président de la République : les vertus éthiques inscrites dans les gestes ne sont ainsi interprétables qu'au regard de pratiques sociodiscursives et de cadres interactifs finement identifiés. Mais cette "inversion corpolectale " nous semble encore illustrer d'une autre manière le caractère social de ces gestes rhétoriques : partagés en une sorte de code ${ }^{16}$, ils ne relèvent pas du corps propre ni d'une gestuelle personnelle; on peut au contraire se les approprier à sa guise, se construire une gestuelle conforme à un modèle socialement proposé, non point «prescrit » bien sûr, mais néanmoins partiellement "pré-écrit»-et c'est sans doute ainsi que les éthè peuvent être intelligibles des auditoires à l'intention desquels ils s'écrivent. C'est donc là une liberté d'usage, non d'innovation, par laquelle chacun peut, dans la limite évidemment de ses moyens personnels (et sur ce plan nous sommes très inégaux), user de son corps comme d'une sorte de palimpseste.

Mais d'un autre côté, il y a à l'évidence une forme de permanence individuelle des pratiques corporelles, qui se routinisent inévitablement et du reste heureusement (on ne réinvente pas sa gestique à chaque fois qu'on agit), et dont on peut penser que chez les professionnels de la communication - comme sont SR et NS - ils ont fait l'objet d'un rodage explicite et sélectif jusqu'à parvenir à ce point où, exactement comme lorsque l'on apprend à écrire, l'instrument est disponible immédiatement et sans peine, sur un mode qui tient à la fois de la maîtrise et du réflexe. Cette appropriation, comparable à un entraînement qui inscrit dans la mémoire corporelle les schémas praxiques les plus efficaces, est bien sûr utile à la réactivité dans les débats : chez nos politiciens comme chez des sportifs, c'est un corps perfectionné qui «répond" comme il a appris à le faire. Elle est également essentielle à la stabilité des éthè : on doit pouvoir, au fil des jours, des mois, des années, reconnaître une personnalité publique comme étant «la même" en dépit de ses évolutions ${ }^{17}$. L'image corporelle elle-même (y compris ses apprêts : coupe de cheveux, vêtements...) y contribue bien sûr largement. Mais les comportements "somato-rhétoriques ${ }^{18}$ " ne sont pas en reste, et ils contribuent également, et puissamment, à l'écriture au long cours des "styles éthiques ». Le style sarkozyen en comporte de nombreux exemples, à inventorier. En voici un, qui illustrera la construction interactive des éthè par la mimogestualité, par le même procédé, à quatre années de distance. La tête inclinée, non pas en mouvement, mais en pose prolongée, avec un petit sourire narquois, n'est pas très caractéristique de ce que nous avions appelé « l'éthos de croisière » sarkozyen (Constantin de Chanay et KerbratOrecchioni, 2007), mais elle compte au nombre de ses armes éthiques dans des occasions bien choisies. Nous l'avons observée ainsi face à J.-M. Le Pen, dont NS réussissait de la sorte à désarçonner la faconde offensive, tout en projetant sur lui l'image d'une sorte de clown, en place du tribun qu'il aurait pu paraître. Et elle réapparaît dans le débat du 2 mai 2007, à un moment bien particulier, l'un des plus conflictuels (colère exceptée, à laquelle la réponse ironique aurait sans doute été insuffisante), pendant lequel au contraire SR, en tous les sens du mot se dresse, avec un port de tête toujours plus droit. Je mets en gras les passages pendant lesquels NS exhibe ce contraste mimo-postural (et prosodique, ce que je ne peux étudier ici), avec donc penchement de la tête et sourire doux : 
$(01.20 .20)$

SR : [...] y a l'problème de crédibilité : voyez-vous parce que quand on a pu: (.)

pendant cinq ans [éventuellement donner une certaine place

NS : [c'est à moi que vous dites ça (.) c'est à moi que vous dites ça

[madame Royal

SR : [donner une certaine place à l'opposition\& NS : c'était pour être désagréable

$\mathrm{SR}$ : \&à l'Assemblée Nationale (.) pourquoi ne l'avez-vous pas [fait\& NS : [mmm

SR : \&vous n'êtes pas crédible [dans la construction de l'État im-

partial

NS : [le problème de crédibilité (.) le

problème de crédibilité vous me le dites comme

[ça

SR : [vous êtes même passé en force avec le

[49.3 donc on en\&

NS : [eh bien dites donc

SR : \&revient [peut-être euh [(disqualifié)\&

AC : [(laissez-la terminer)

PPDA : [allez on on parle des impôts s'il vous plaît

Dans le détail, le contraste prend bien sûr un tout autre sens que face à J.-M. Le Pen : ici, on peut voir une stratégie sexiste dans le fait de transposer sur le plan affectif (et pour tout dire, presque nunuche), des reproches relevant pour son interlocutrice du plan politique. Ce faisant, tout en construisant propre éthos, NS construit par conséquent aussi celui de SR, lui parlant non comme à une adversaire qui argumente contre lui, mais comme à une sorte d'aimable mégère qui eût laissé percer dans le sérieux du débat médiatique une manifestation d'humeur bien féminine et dont il serait permis, voire opportun ( $\mathrm{du}$ fait de son caractère déplacé), de se gausser légèrement avec la connivence de l'auditoire. À quatre ans de distance, avec des effets de sens certes différents puisque les interactions concernées sont spécifiques, mais inscrit dans une stratégie argumentative identique (utiliser un contraste postural pour infléchir négativement l'image de l'interlocuteur), nous retrouvons le même geste d'écriture somato-rhétorique. Parler de l'actio rhétorique comme d'une corpographèse n'est donc pas un vain mot: ce que le corps écrit dans l'interaction par l'action hic et nunc de sa propre matière, se grave en retour en lui et s'inscrit au nombre de ses compétences rhétoriques «stabilisées » et d'autant plus facilement disponibles - les écrits restent.

\section{BIBLIOGRAPHIE}

Amossy, R. (dir.), 1999, Images de soi dans le discours. La construction de l'éthos, Lausanne, Delachaux et Niestlé.

Aristote, 1991, Rhétorique, Paris, UGE, coll. « Le Livre de Poche ».

Berrendonner, A., 1981, Éléments de pragmatique linguistique, Paris, Minuit.

Bouvet, D., 2001, La Dimension corporelle de la parole. Les marques posturo-mimo-gestuelles de la parole, leurs aspects métonymiques et métaphoriques et leurs rôles au cours d'un récit, Paris, Peeters. 
Bres, J., Haillet, P.-P., Mellet, S., Nølke, N. et Rosier, L. (dir.), 2005, Dialogisme et Polyphonie : approches linguistiques, actes du colloque de Cerisy, 3-9 septembre 2004, Bruxelles, De BoeckDuculot.

Calbris, G., 2003, L'Expression gestuelle de la pensée d'un homme politique, Paris, CNRS Éditions.

-, 2008, « La Tête de Nicolas Sarkozy », Mots, $n^{\circ}$ 86, février 2008, p. 101-118.

Charaudeau, P., 2005, Le Discours politique. Les masques du pouvoir, Paris, Vuibert.

Constantin de Chanay, H. et Le Guern, O., à paraître, « Éthos en pâture : images et contre-images de Nicolas Sarkozy ", Actes des XII ${ }^{e}$ Journées internationales de sémiotique : Information, propagande et opinion (par ou avec les images), 20 et 21 octobre 2007, rencontres proposées par le groupe EIDOS, sous la direction de Michel Costantini (Paris 8).

Constantin de Chanay, H. et Kerbrat-Orecchioni, C, 2007, « 100 minutes pour convaincre : l'éthos en action de Nicolas Sarkozy ", dans M. Brothet et al. (dir.), Le Français parlé des médias, Stockholm, Acta Universitatis Stokholmiensis, p. 309-329.

Cosnier, J., Coulon, J., Berrendonner, A., et Orecchioni, C, 1982, Les Voies du langage. Communications verbales, gestuelles et animales, Paris, Dunod.

Cosnier, J. et Brossard, A., 1984, La Communication non verbale, Neuchâtel, Delachaux et Niestlé.

Cosnier, J., 2000, « Définition et conception du non verbal pour le "psychologue" », Communication et Organisation, $\mathrm{n}^{\circ} 18$, Bordeaux, ISIC, p. 49-55.

Ducrot, O., 1984, Le Dire et le Dit, Paris, Minuit.

Kerbrat-Orecchioni, C, 2000 : « Rhétorique et interaction », in R. Koren et R. Amossy (dir.), 2000, Après Perelman : quelle politique pour les nouvelles rhétoriques?, Paris, L’Harmattan.

-, 2004, «Que peut-on "faire" avec du dire ? ", Cahiers de linguistique française, $\mathrm{n}^{\circ}$ 26, p. 27-43.

Kraft, U. et Dausendschön-Gay, U., 2001, « La multidimensionnalité de l'interaction. Textes, gestes, et le sens des actions sociales ", Marges Linguistiques, nº 2, nov. 2001, p. 120-139.

Nølke, H., 2001, Le Regard du locuteur II, Paris, Kimé.

Perrin, L., 2004, « Le Discours rapporté modal », dans J. Lopez-Muñoz, S. Marnette et L. Rosier (dir.), Le Discours rapporté dans tous ses états, Paris, L'Harmattan, p. 64-74.

-, 2006 (dir.), Le Sens et Ses Voix. Dialogisme et polyphonie en langue et en discours, $\mathrm{n}^{\circ} 28$ de Recherches Linguistiques, Metz, université de Metz.

Sapir, E., 1967, Anthropologie, Paris, Minuit.

Scollon, R. et Scollon, S., 1995, « Somatic Communications: How Useful is "Orality" for the Characterization of Speech Events and Cultures? », dans U. M. Quasthoff (dir.), Aspects of Oral Communication, Berlin et New York, W. de Gruyter, p. 19-30.

Wittgenstein, L., 1961 [1921], Tractactus logico-philosophicus, Paris, Gallimard, coll. « Idées ».

\section{NOTES}

1. D'une durée totale de $2 \mathrm{~h} 40$, ce débat diffusé simultanément sur TF1 et sur France 2 était animé par Arlette Chabot et Patrick Poivre d'Arvor. Nous désignerons désormais les quatre protagonistes par leurs initiales, à savoir SR et NS pour les deux candidats à l'élection présidentielle, AC et PPDA pour les deux journalistes. 
2. Elle l'est encore, à l'adresse: http://www.liberation.fr/actualite/politiques/ elections2007/251273.FR.php (dernière consultation le 14 avril 2008) [NDLR: aujourd'hui disponible à l'adresse suivante: http://www.liberation.fr/cahier-special/2007/05/03/sarkozyroyal-le-debat-12_92063 (juin 2014)]. Un simple décompte montre l'ampleur de la réécriture de l'oral que réalisent des transcriptions « toilettées ", même quand elles cherchent à rester proches de la spontanéité des interventions : la transcription des journalistes compte un peu moins de 150000 signes, ce qui correspond à un peu moins de 25000 mots. Ma propre transcription du même débat compte près de 230000 signes, et 38500 mots, au nombre desquels les reprises, inachèvements, hésitations, etc., si caractéristiques de la parole fluide qu'elles contribuent à lubrifier et à rendre "vivante ", mais extrêmement pénibles à la lecture d'un texte présenté sous forme écrite. Si l'on ajoute à cela les mimiques, gestes, postures sur lesquels nous allons nous concentrer et qui sont évidemment absents de la transcription, on mesure l'écart considérable qui sépare le débat que l'on peut lire de celui que l'on peut visionner.

3. Laquelle détaille plusieurs étapes d'élaboration du discours : inventio, dipositio, elocutio, et actio, cette dernière consistant en une prestation orale réclamant, comme pour les acteurs, le concours du corps (voix et gestes).

4. Pour plus de détails sur la notion d'éthos, voir en particulier Amossy (1999); et sur les réaménagements dont il est question ici, je me permets de renvoyer à Constantin de Chanay et Kerbrat-Orecchioni (2007), ainsi qu'à Constantin de Chanay et Le Guern (à paraître).

5. Élargissement déjà pratiqué dès l'Antiquité : comme me le fait remarquer P. Zoberman, «les parlementaires $d u \mathrm{XVII}^{\mathrm{e}}$ siècle présentent, en s'appuyant sur Cicéron, des portraits contrastés d'orateurs, positifs ou négatifs, sur le plan de l'éthos » (communication personnelle).

6. Le chapitre entier a pour titre « L'illusion interrogative »...

7. Comme Berrendonner, j'utilise l'opposition wittgensteinienne (Tractatus, 4.022) entre dire et montrer. Pour faire bref, je les opposerai en fonction de deux axes: (i) la sensibilité à la véridiction, ce qui est dit étant toujours contestable, tandis que le montré, seulement constatable, est d'emblée soustrait au débat (critère issu de Nølke $2001: 81$ ) ; (ii) la solidarité avec un hic et nunc énonciatif, le dit s'en émancipant, tandis que le montré y est indéfectiblement attaché, d'où sa valeur indicielle symptomatique (critère issu de Berrendonner). Comme Nølke je considère qu'il s'agit là des deux opérations énonciatives primitives. Si l'on suit Berrendonner, on peut sans doute les subordonner l'une à l'autre : le dire est ancré dans le montrer (ce qui fait de la " corpographèse » le socle de la parole).

8. Débat diffusé sur BFM TV et actuellement visible intégralement sur YouTube. [NDLR: Aujourd'hui disponible sur le site de l'INA : http://www.ina.fr/video/3341956001/2007-le-debatsegolene-royal-et-nicolas-sarkozy-video.html (juin 2014).]

9. Polyphonie/dialogisme : les termes ne sont pas équivalents pour tout le monde, sans qu'il y ait de définition consensuelle, si ce n'est que tous deux désignent des phénomènes d'hétérogénéité énonciative au sein d'un même discours. Personnellement je tends à utiliser dialogisme dans les cas de citation, d'allusion, de discours représentés, etc., qui font intervenir symboliquement d'autres locuteurs; et polyphonie dans les cas de superposition de points de vue qui ne sont pas forcément attribuables à des instances réelles, mais que la structure de l'énoncé interdit de cumuler sur une seule source énonciative, par exemple un point de vue et son contraire dans le cas d'un énoncé négatif - que l'on rattache à des positions abstraites, que les « énonciateurs » de Ducrot (1984), qui ne sont pas des locuteurs, servent uniquement à contraster, nullement à incarner.

10. Outre nos contributions dans ces deux volumes, citons dans Bres et al. (2005) les études de Barberis et de Vérine, et dans Perrin (2006) celle de Auchlin et Grobet.

11. Souvent accompagné de celui du haut du buste et des épaules, solidaires - on peut d'ailleurs dire qu'en général, dans sa gestuelle vive et très fluide, l'ensemble du corps suit ou accompagne le mouvement du ou des segment(s) corporel(s) qui sont le(s) site(s) du ou des geste(s) - car il 
n'est pas rare, loin de là, qu'il y ait plusieurs gestes concomitants -, en une sorte de "danse » globale assez homogène.

12. Autres conventions de transcription particulières : / = intonation montante $\backslash \backslash=$ intonation descendante $;($.$) = pause ;[\mathrm{xxx}]$ = séquence en chevauchement $;(\mathrm{xxx})=$ séquence incertaine $; \&=$ continuité du tour de parole; $\rightarrow=$ changement de tour intra-locuteur, en réaction immédiate à un chevauchement, sans interruption du flux de parole ; $\neq=$ changement de tour intra-locuteur et intra-réplique, c'est-à-dire réaction différée, en milieu de réplique, à une intervention précédente ou à un chevauchement.

13. Par contre, une négation gestuelle n'annule pas une négation linguistique, ce que je ne peux développer ici. Voir sur ce point, par exemple, Calbris 2008.

14. La langue française permet de distinguer entre ce qui relève du niveau énonciatif des positions discursives hétérogènes (le «dialogique ») et ce qui relève du niveau interlocutif des participants (le « dialogal »).

15. Je ne veux pas dire par là que $S R$ ne manie pas, comme tout un chacun, la polyphonie. Simplement, dans le cadre précis de ce débat, elle le fait de manière beaucoup moins diversifiée que son adversaire.

16. Code " écrit nulle part ", dit Sapir dans un texte célèbre (1967:46) - mais à un autre niveau, ces pratiques ont bien toute la permanence des archives.

17. Le célèbre «j'ai changé » de NS eût été dangereux s'il n'avait pas été interprété comme une amélioration, c'est-à-dire un /je suis devenu (encore) plus proche de ce que je suis idéalement/.

18. J'entends donc par là tout autre chose que les « tics » dont les imitateurs sont friands.

\section{RÉSUMÉS}

Cet article a pour but d'évaluer à quel point le corps considéré dans une perspective interactive et multimodale joue un rôle fondamental (par le biais de gestes, de postures, de mimiques et de regards) dans la construction de positions d'énonciation et d'identités discursives (éthos) dans les débats se déroulant dans les médias politiques et précisément lors du débat de mai 2007 opposant Nicolas Sarkozy (NS) à Ségolène Royal (SR). NS peut physiquement composer une image somatique de lui-même, démontrant par l'action sa capacité à unir son public diversifié (ce qui lui donne une longueur d'avance dans le contexte d'une élection présidentielle) par opposition à SR, qui s'apparente davantage à une activiste dont le discours semble destiné à son propre électorat traditionnel. Le corps est un instrument que l'on utilise pour « écrire ", dans l'espace et de façon dynamique, des identités dans l'interaction en cours, aussi bien qu'un matériau brut, pour ainsi dire, et le médium d'écriture durable des identités même qu'il contribue à définir.

This article aims to assess how fondamental a role the body, viewed in an interactive and multimodal perspective, plays (through gestures, postures, mimics, and glances) in the construction of positions of enunciation and discursive identities (ethos) in political media debates and specifically the May 2007 debate berween Nicolas Sarkozy (NS) and Ségolène Royal (SR). NS can physically compose a somatic image of himself, demonstrating in action his capacity to unite his diverse audience-a definite edge in the context of a presidential election-in contrast with SR, who appears more as an activist whose discourse seems geared toward her own traditional electorate. The body is both an instrument used to "write," spatially and dynamically, 
identities in the ongoing interaction, as well as the raw material, so to speak, and the médium for durably writing the very identities it contributes to define.

INDEX

Mots-clés : éthos, polyphonie, énonciation, gestes, regards

Keywords : ethos, polyphony, enunciation, gestures, glances

\section{AUTEUR}

\section{HUGUES DE CHANAY}

Université Lyon 2 - Louis-Lumière 